\title{
Assessing the Beneficial Impact of a Patient Support Program in Secukinumab-Treated Patients with Psoriasis in Italy
}

Giuseppe Argenziano,' '* Paolo Amerio, (ID) 2 Maria Grazia Aragone, ${ }^{3}$ Ginevra Baggini, ${ }^{4}$ Manuela Baldari, ${ }^{5}$ Gianpiero Castelli, ${ }^{6}$ Simmaco Coppola, (iD) Antonio Costanzo, ${ }^{8,9}$ Rocco De Pasquale, ${ }^{10}$ Maria Concetta Fargnoli, (ID ${ }^{11}$ Caterina Foti, ${ }^{12}$ Claudia Giofrè, ${ }^{13}$ Serena Lembo, ${ }^{14}$ Claudia Leporati, ${ }^{15,16}$ Francesco Loconsole, ${ }^{17}$ Giovanna Malara, ${ }^{18}$ Ketty Peris, ${ }^{19,20}$ Antonio Giovanni Richetta, (ID) ${ }^{21}$ Paola Savoia, (ID ${ }^{22}$ Rossana Tiberio, ${ }^{23}$ Massimo Travaglini, (ID ${ }^{24}$ Maria Teresa Uzzauto, ${ }^{25}$ Luca Bianchi ${ }^{26, *}$

'Dermatology Unit, University of Campania, Naples, Italy; ${ }^{2}$ Department of Medicine and Aging Science and Dermatologic Clinic, University "G. d'Annunzio", Chieti-Pescara, Chieti, Italy; ${ }^{3} \mathrm{ASL}$ AL, Alessandria, Italy; ${ }^{4} \mathrm{Osp}$. Civile di Varzi, Pavia, Italy; ${ }^{5}$ ASL4 Liguria, Genova, Italy; ${ }^{6}$ UOSD of Dermatology, Ospedale Umberto I ${ }^{\circ}$, Siracusa, Italy; ${ }^{7}$ IQVIA, Rome, Italy; ${ }^{8}$ Department of Biomedical Sciences, Humanitas University, Milano, Italy; ${ }^{9}$ Skin Pathology Laboratory, Humanitas Research Hospital IRCCS, Milano, Italy; ${ }^{10} \mathrm{UOC}$ Dermatologia, AOU Policlinico "G. Rodolico San Marco", Catania, Italy; " Dermatology, Department of Biotechnological and Applied Clinical Sciences, University of L'Aquila,

L'Aquila, Italy; ${ }^{12}$ Department of Biomedical

Science and Human Oncology, Dermatological

Clinic, University of Bari, Bari, Italy; ${ }^{13}$ U.O.C.

Dermatologia, A.O. Papardo, Messina, Italy;

${ }^{14}$ Dipartimento di Medicina, Chirurgia e

Odontoiatria Scuola Medica Salernitana,

University of Salerno, Fisciano, Italy;

${ }^{15}$ Dermatology Unit, ASL AL Casale

Monferrato, Alessandria, Italy; ${ }^{16}$ Dermatology

Unit, ASL TO4 Ivrea, Torino, Italy;

${ }^{17}$ Department of Dermatology, University of

Bari, Bari, Italy; ${ }^{18}$ UOC of Dermatology,

Grande Ospedale Metropolitano "BMM",

Reggio Calabria, Italy; ${ }^{19}$ Institute of

Dermatology, Catholic University, Rome, Italy;

${ }^{20}$ Dermatology Unit, Fondazione Policlinico

Universitario A. Gemelli IRCCS, Rome, Italy;

${ }^{21}$ Dipartimento di Scienze Cliniche

Internistiche e Cardiovascolari Policlinico

Umberto I University of Rome "La Sapienza",
Purpose: For patients with psoriasis, treatment adherence and persistence are fundamental if therapeutic goals are to be met. Patient Support Programs (PSPs) may be used as a support tool to assist patients and health care professionals optimize treatment and improve disease management.

Patients and Methods: In Italy, the PSP PSOLife CARE, which began on the 9th of February 2017 and is ongoing, aimed to support patients with psoriasis under therapy with secukinumab (Cosentyx ${ }^{\circledR}$ ). A team of medical professionals including Dermatologists, Psychologists, Nutritionists, and field Nurses provided outpatient treatment as well as remote support via phone calls. Patients had a standard duration in the Program of 6 months. This report analyzes the data of patients who benefited from the Program from February 2017 to August 2020, for a total observation of 42 months.

Results: We provide here a descriptive report on the benefits of participation in the PSOLife CARE Program for patients with psoriasis and medical professionals involved in their care. Throughout their time in the PSOLife CARE Program, patient satisfaction remained consistently high with sustained improvements observed in all aspects of quality of life (ie emotional, social, physical, and economic). Despite exiting from the Program, most patients continued to adhere to secukinumab. Medical professionals also reported positive outcomes on their interactions with patients, with more than half of those surveyed rating the overall quality of the Program as "Outstanding".

Conclusion: By supporting treatment adherence, the PSOLife CARE Program may have empowered patients to better manage their psoriasis, increasing their satisfaction with treatment and quality of life.

Keywords: dermatology, PSOLife CARE Program, quality of life, treatment adherence

\section{Plain Language Summary}

- Psoriasis is a chronic, immune-mediated, inflammatory skin condition characterized by red, scaly patches with symptoms of itching, burning, and pain. For people with psoriasis, the condition can be debilitating and severely affect everyday living, causing a considerable burden.

- Biological therapies, which target specific components of the immune system, are very effective treatments for psoriasis. However, adherence to and persistence with prescribed therapies are fundamental if therapeutic goals are to be met.

- To improve treatment adherence, pharmaceutical companies may offer Patient Support Programs to augment therapeutic strategies and improve patient outcomes through better disease management. 
Rome, Italy; ${ }^{22}$ Department of Health Science, Università del Piemonte Orientale, Novara, Italy; ${ }^{23}$ SCDU Dermatologia, AOU Maggiore della Carità, Novara, Italy; ${ }^{24}$ U.O.S.D. dermatologica - centro per la cura della psoriasi, Ospedale Perrino, Brindisi, Italy; ${ }^{25}$ U.O.C. Dermatologia, ASL Salerno, Ospedale "A:Tortora"-Pagani, Salerno, Italy; ${ }^{26}$ Dermatology Unit, Tor Vergata University Hospital, Rome, Italy

*These authors contributed equally to this work
Correspondence: Giuseppe Argenziano

Dermatology Unit, University of Campania "Luigi Vanvitelli", Via

Pansini, 5, Naples, 80I3I, Italy

Tel +393354l5093

Fax +39069762 5822

Email g.argenziano@gmail.com

Luca Bianchi

University of Rome "Tor Vergata",

Policlinico Tor Vergata, Viale Oxford

81 , Rome, 00133 , Italy

Tel +390620902739

Fax +390620902742

Email luca.bianchi@uniroma2.it
- The Italian PSOLife CARE Patient Support Program provided in-person and remote support for patients with psoriasis under therapy with secukinumab (known as Cosentyx ${ }^{\circledR}$ ) through a dedicated team of Dermatologists, Psychologists, Nutritionists, and field Nurses.

- Throughout the PSOLife CARE Program, patient satisfaction remained consistently high with sustained improvements observed in all aspects of daily life.

- Most people who took part in the PSOLife CARE Program continued treatment with secukinumab despite exiting from the Program.

○ Health care professionals also felt supported and reported positive outcomes with their patients.

- Overall, the PSOLife CARE Program improved adherence to secukinumab, increasing treatment satisfaction. Ideally, this should motivate patients to better manage their psoriasis leading to them having a more positive outlook of their condition and therapeutic regimen.

\section{Introduction}

Psoriasis is a chronic, immune-mediated, inflammatory skin disease affecting $2 \%$ to $4 \%$ of the Western population. ${ }^{1}$ Its pathophysiology is complex and associated with the dysregulation of the immune system. In Italy, the reported prevalence of psoriasis has been estimated at $1.8 \%$ to $3.1 \%$, with high variability (from $0.8 \%$ to $4.5 \%$ ) across the regions. ${ }^{2-4}$ In 2010 , an incidence rate of 2.30 to 3.21 cases per 1000 person-years was calculated from an Italian study cohort of 511,532 adults who had received a first-ever diagnosis of psoriasis from 2001 to $2005 .^{5}$

The clinical manifestations of psoriasis vary between individuals. Plaque psoriasis is the most common form, typically presenting as well-defined, scaly erythematous patches or plaques, and occurring in $85 \%$ to $90 \%$ of affected patients. ${ }^{1}$ Symptoms of psoriasis include itching, burning, and pain. Psoriasis is commonly associated with physical and psychological comorbidities, which, together with symptoms, can negatively affect quality of life and work productivity causing considerable burden to the patient. ${ }^{6}$

Patients with mild-to-moderate psoriasis can be treated with topical agents such as glucocorticoids, vitamin D analogs, and phototherapy; patients with moderate-to-severe psoriasis may require conventional or biologic systemic treatments. $^{7}$ Novel biologic therapies, which target specific inflammatory pathways including the tumor necrosis factor- $\alpha$ signaling pathway and the interleukin (IL)-23/TH17 axis, have improved treatment outcomes without compromising patient safety. ${ }^{8}$ Secukinumab (Cosentyx ${ }^{\circledR}$ ), a fully human monoclonal antibody, selectively targets IL-17A and has long-lasting efficacy in the treatment of psoriasis as well as a favorable safety profile. ${ }^{9-15}$ It is approved in Europe and the USA for the treatment of moderate-to-severe plaque psoriasis in adults who are candidates for systemic therapy at a recommended dose of 300 $\mathrm{mg}$ by subcutaneous injection at weeks $0,1,2,3$, and 4, followed by $300 \mathrm{mg}$ every 4 weeks thereafter. ${ }^{16,17}$

If therapeutic goals are to be met for patients with psoriasis, they must adhere to the prescribed treatment regimen. However, like all drugs, there is always a theme of non-adherence to treatment for various reasons. It could be argued that patients with a chronic inflammatory disease such as psoriasis are a more "fragile 
population" who need greater attention. These "fragile" patients may be insecure in the management of selfadministration of biological drugs due to the need to inject the drug (ie, to understand in-depth how the drug is administered, how to use the device, and the areas where the drug is injected), and who need reminding of specialist's recommendations, follow-up visits, and examinations. In addition, when the lesion has disappeared, patients often tend to try to "skip" administration of their prescribed treatment regimen, considering themselves "healed". Only the clinician can decide on any change or suspension of therapy because, due to the chronicrelapsing nature of psoriasis, the disease never heals but can be kept under control over time with appropriate drugs. Patient Support Programs (PSPs), which assist patients and health care professionals to optimize treatment and improve disease management, have increased treatment adherence and persistence across a broad range of autoimmune diseases including psoriasis. ${ }^{18-20}$ Indeed PSPs, address all the issues mentioned above to ensure patients are autonomous, safe, aware, and have improved treatment adherence.

The PSP PSOLife CARE, funded by Novartis, designed and executed by IQVIA, aimed to support patients with psoriasis in Italy under therapy with secukinumab. This PSP was implemented at no cost to patients or the Italian National Health Services. The PSOLife CARE Program, which began on the 9th of February 2017 and is ongoing, provides in-person and remote support to patients and involves a team of medical professionals including Dermatologists, Psychologists, Nutritionists, and field Nurses.

Herein is a descriptive report on the relationship between participation in the PSOLife CARE Program and patient satisfaction with treatment, quality of life, and treatment adherence, as well as the impact of the PSP on the satisfaction and approach of medical professionals involved in the care of patients with psoriasis in Italy.

\section{Methods}

\section{Setting}

This is a descriptive report of the PSOLife CARE Program for secukinumab-treated patients with psoriasis in Italy. Clinical Centers distributed throughout Italy were invited to take part in the Program and patients were recruited from February 2017. This report analyzes the data of patients who benefited from the Program from February 2017 to August 2020, for a total observation of 42 months. Physicians of activated Clinical Centers presented the PSP to eligible patients under treatment with secukinumab; patients were free to choose whether or not to be enrolled in the Program. All data were collected by telephone, directly from the patients. The questionnaires used are standard, and the data recorded on a dedicated platform. All patients gave written informed consent to participate in the PSP. Due to the nature of the PSOLife CARE Program, ethical approval was not required because it is not a clinical study. Instead, PSPs are a category of projects that support patients in managing their therapy/pathology. They are not defined as clinical studies as their activities do not endorse a new drug, observe a phenomenon, or compare different patient populations in therapy with different products. Instead, PSPs offer services that promote better patient adherence to the therapy assigned by their doctor.

PSPs, of course, must comply with the rules on Pharmacovigilance and Privacy, with appropriate procedures and forms in place to permit full compliance. For the PSOLife CARE, the following is envisaged:

1. The adhesion by the responsible doctor by filling in the form and privacy information (with specific, explicit purpose: "To carry out anonymous statistical surveys and scientific publications");

2. The doctor, starting from his adhesion, can present the PSP to the patients in therapy;

3. Patients can join the PSP by filling in the form and privacy information (with specific, explicit purpose: "To carry out anonymous statistical surveys and scientific publications");

4. IQVIA collaborators collect documents on the territory while carrying out the planned activities.

For PSPs, there is no approval flow from the ethics committee of each Center, as there is no protocol (or similar) to view. Instead, the supporting material merely details the type of services provided and available to patients. Furthermore, from a "legislative" point of view, the only framework that regulates PSPs is the Farmaindustria code (Point 4.7), which details the above and does not provide any approval flow for the aforementioned programs.

\section{Data Collection}

As this was not a clinical trial, there were no specific inclusion or exclusion criteria, except that patients had to be affected by psoriasis and treated with secukinumab. 
Data were collected regarding the number of months between the date patients first received secukinumab (Therapy start date) and the date patients were enrolled in the Program (Program start date) during the different Program periods. Therapy administration dates, as described below, and the date of therapy discontinuation were also collected.

Patients had a duration in the Program of 6 months; measurements were also taken at 12 and 24 months for a limited number of patients. Figure 1 depicts the Program structure through which a patient journeyed showing the number of times a patient was surveyed during their time in the Program and after their exit from the Program, as well as optional visits to medical personnel.

The dates of secukinumab administration were collected during the 6 months of the Program in order to assess management of therapy administrations. Data were assessed as to whether secukinumab had been administered in an acceptable or anomalous range according to the recommended dosing schedule (ie $300 \mathrm{mg}$ by subcutaneous injection at weeks $0,1,2,3$, and 4 , followed by $300 \mathrm{mg}$ every 4 weeks thereafter). For the first 4 weeks in the Program, dosing in a range of 0-9 days was considered acceptable whereas a range of 10-23 days was considered anomalous. Similarly, for dosing every 4 weeks thereafter, a range of 24-34 days was considered acceptable and $\geq 35$ days was considered anomalous.

Patients were treated as outpatients and data on their adherence to treatment, quality of life, and the number of outpatient visits were collected. Patients were also provided with remote support, education, and training via phone calls.

At each Clinical Center, patients were overseen by one or more of the following medical professionals: Dermatologist, Nutritionist, Psychologist, and/or field Nurse. The monthly distribution and type (Dermatologic, Nutritional, Psychological, or field Nurse) of visits and phone calls were recorded.

\section{Questionnaires}

Patients were asked to express his/her opinion on their overall satisfaction with the PSOLife CARE Program, assessed using a score of 1 to 5 (1 - Did not meet my expectations; 2 - Fairly Satisfactory; 3 - Satisfactory; 4 Very Satisfactory; 5 - Outstanding), and completed a 6question survey detailing their satisfaction (Table 1). This survey was designed to assess the quality of the Program and of information shared, the usefulness of the Program on the management of visits as well as on therapy management and pathology management, and whether the patients felt the Program should be changed. The patient satisfaction questionnaire was administered twice per patient during the Program.

The impact of psoriasis on patients' quality of life was assessed at months 1-6, 12, and 24 using a 4-question survey. In this survey, patients were asked to evaluate the impact of their psoriasis on the emotional, social, physical, and economic aspects of daily life, each with a score between 0-100, where " 0 " means a low impact and "100" means a high impact.

PSOLife CARE Program

EXIT

\begin{tabular}{|c|c|c|c|c|c|c|c|}
\hline Month 1 & Month 2 & Month 3 & Month 4 & Month 5 & Month 6 & Month 12 & Month 24 \\
\hline $\begin{array}{c}\text { Adherence, } \\
\text { QOL, } \\
\text { Satisfaction }\end{array}$ & $\begin{array}{c}\text { Adherence, } \\
\text { QOL }\end{array}$ & $\begin{array}{l}\text { Adherence, } \\
\text { QOL, } \\
\text { Satisfaction }\end{array}$ & $\begin{array}{c}\text { Adherence, } \\
\text { QOL }\end{array}$ & $\begin{array}{c}\text { Adherence, } \\
\text { QOL }\end{array}$ & $\begin{array}{c}\text { Adherence, } \\
\text { QOL }\end{array}$ & $\begin{array}{c}\text { Adherence, } \\
\text { QOL }\end{array}$ & $\begin{array}{c}\text { Adherence, } \\
\text { QOL }\end{array}$ \\
\hline
\end{tabular}

\begin{tabular}{|ll|}
\hline Dermatologist visit (optional) & $\times 3$ times \\
Psychologist visit (optional) & $\times 2$ times \\
Nutritionist visit (optional) & $\times 2$ times \\
Nurse visit (optional) & $\times 3$ times \\
\hline
\end{tabular}

Figure I PSOLife CARE program structure. Abbreviation: QOL, quality of life. 
Table I Patient Satisfaction Questionnaire

\begin{tabular}{|l|l|}
\hline Question & Response \\
\hline 1). Have you been advised with clear and fully explained information about the services provided within the Program to easily activate them? & Score a \\
2). How do you define the quality of the Program? & Score a \\
3). Do you feel more confident in drug administration and management thanks to this Program? & Score $^{\text {a }}$ \\
4). Do you find this Program helpful in following the Clinical Centre's recommendations? & Score ${ }^{\text {a }}$ \\
5). Do you feel supported by this Program in dealing with the management of your psoriasis? & Score ${ }^{\text {a }}$ \\
6). Do you think the Program should be changed in some way? & Yes/No \\
\hline
\end{tabular}

Notes: ${ }^{\mathrm{a}}$ I - Did not meet my expectations; 2 - Fairly Satisfactory; 3 - Satisfactory; 4 - Very Satisfactory; 5 - Outstanding.

Medical professionals, with patients who had $>6$ months duration in the Program, were invited to complete a Satisfaction Questionnaire on the PSOLife CARE Program to evaluate the impact of the Program on their approach with patients (Table 2).

To assess the usefulness of the Program for ongoing adherence to therapy, patients were surveyed at 12 months and 24 months (Table 3). In this survey, patients were questioned on the long-term impact of the PSP and whether they felt the Program had been beneficial for their ongoing therapy adherence. Patients were also questioned as to whether they were still under treatment with secukinumab and, if not, were questioned as to why.

The questionnaires used to assess patient satisfaction, medical professional satisfaction, the impact of psoriasis on patients' quality of life, and patient adherence to

Table 2 Medical Professional Satisfaction Questionnaire

\begin{tabular}{|c|c|}
\hline Question & Response \\
\hline I). Do you find this Program the most suitable solution to deal with patients in treatment with secukinumab? & Score ${ }^{a}$ \\
\hline \multicolumn{2}{|l|}{ 2). In which of the following aspects do you feel more supported? } \\
\hline a. Is the patient more followed and aware? & Yes/No \\
\hline b. Are the monitoring visits taking less time? & Yes/No \\
\hline c. Could you get more information about the patients' disease management? & Yes/No \\
\hline d. Any other relevant aspect? & Yes/No \\
\hline \multicolumn{2}{|l|}{ 3). If Yes to question 2a, in which aspect do you find the patient more followed and aware? } \\
\hline a. Management of secukinumab & $\%$ \\
\hline b. Management of side effects & $\%$ \\
\hline c. Management of the dermatological aspect & $\%$ \\
\hline d. Management of the psychological aspect & $\%$ \\
\hline e. Management of the nutritional aspect & $\%$ \\
\hline f. Others & $\%$ \\
\hline \multicolumn{2}{|l|}{ 4). If Yes to question $2 b$, what is the average time saved at each monitoring visit? } \\
\hline a. $<5$ minutes & $\%$ \\
\hline b. $5-10$ minutes & $\%$ \\
\hline c. $10-20$ minutes & $\%$ \\
\hline d. $>20$ minutes & $\%$ \\
\hline \multicolumn{2}{|l|}{ 5). If Yes to question $2 c$, which kind of information did you get thanks to the Program? } \\
\hline a. Patient adherence & $\%$ \\
\hline b. Compliance with the recommendations & $\%$ \\
\hline c. Side effects & $\%$ \\
\hline d. Other? If yes, which aspects could you mention? & $\%$ \\
\hline \multicolumn{2}{|c|}{ 6). If Yes to question 2d, which kind of information could you get thanks to the Program in addition to the ones yet mentioned? } \\
\hline 7). What is your opinion about the overall quality of the Program? & Score ${ }^{a}$ \\
\hline
\end{tabular}

Notes: ${ }^{\mathrm{a}}$ - Did not meet my expectations; 2 - Fairly Satisfactory; 3 - Satisfactory; 4 - Very Satisfactory; 5 - Outstanding. 
Table 3 Patient Adherence Questionnaire

\begin{tabular}{|c|c|}
\hline Question & Response \\
\hline $\begin{array}{l}\text { I. After some time out of the Program, in which of the following aspects do you think the PSOLife Program's calls have been helpful? } \\
\text { a. Therapy Adherence } \\
\text { b. Organization/Reminder of the visits } \\
\text { c. Emotional Support }\end{array}$ & $\begin{array}{l}\% \\
\% \\
\%\end{array}$ \\
\hline $\begin{array}{l}\text { 2. How do you consider/assess your therapy adherence since you are no longer under the Program? } \\
\text { a. Excellent }(>95 \%) \\
\text { b. Good }(>80 \% \text { to }<95 \%) \\
\text { c. Low }(<80 \%)\end{array}$ & $\begin{array}{l}\% \\
\% \\
\%\end{array}$ \\
\hline 3. Are you still under treatment with secukinumab? & Yes/No \\
\hline $\begin{array}{l}\text { 4. If No, specify the motivation } \\
\text { a. Not needed } \\
\text { b. Switch to a similar biologic drug } \\
\text { c. Change of therapy }\end{array}$ & $\begin{array}{l}\% \\
\% \\
\%\end{array}$ \\
\hline
\end{tabular}

treatment were created specifically for the PSOLife CARE Program and have not been validated in other support programs.

\section{Data Analysis}

Data are presented as whole numbers, cumulative numbers, or percentages. Data analysis used a cumulative method with a weighted average for the calculation of the average satisfaction rate. The data of quality of life and secukinumab administration date are aggregated and analyzed with average by month of the patient's stay in the program.

\section{Results}

\section{Patient Support Program Services}

Clinical Centers were distributed throughout Italy with a total of 28 active centers. Of these, 6 Clinical Centers located in Northern Italy, 5 in Central Italy, and 9 in Southern Italy had patients.

The availability of a Dermatologist, Psychologist, Nutritionist, or field Nurse varied amongst Centers. Except for a center in Central Italy, all Centers had a field Nurse. A Dermatologist was present in all Northern and Central Centers and 6 of the 9 Centers in Southern Italy. A Psychologist was available in 3, 1, and 7 Centers in North, Central, and South Italy, respectively; 3 Centers in Northern Italy and 2 in Southern Italy had a Nutritionist.

The uptake of patients into the PSOLife CARE Program was distributed over 42 months, culminating in a total uptake of 525 patients (Figure 2). There was a noticeable decrease in the uptake of patients into the Program from February 2020.

The average patient age was 51.45 years and $62.4 \%$ (327/524; this information was not available for 1 patient) of the patients were male. The average time period between the Therapy start date and Program start date ranged from 0.6 months to 5.7 months (Table 4). From October 2019 onwards, the average time period between Therapy and Program start dates was noticeably less than at the start of the Program, which was due to two principal reasons. From October 2019, most of the targeted Clinical Centers had been activated and, in the first phase (after activation), the Clinical Centers already had patients in therapy. Therefore, after the start-up phase, the Clinical

Table 4 Average Time Period in Months Between the Date Patients First Received Secukinumab (Therapy Start Date) and the Date Patients Were Enrolled in the Program (Program Start Date)

\begin{tabular}{|l|c|c|}
\hline & $\begin{array}{c}\text { Time Period, } \\
\text { Months }\end{array}$ & $\begin{array}{c}\text { No. of } \\
\text { Patients }^{\mathbf{a}}\end{array}$ \\
\hline April 2017-September 2017 & 5.7 & 69 \\
October 2017-March 2018 & 3.4 & 108 \\
April 2018-September 2018 & 4.2 & 79 \\
October 2018-March 2019 & 5.3 & 133 \\
April 2019-September 2019 & 3.4 & 75 \\
October 2019-March 2020 & 0.7 & 39 \\
April 2020-September 2020 & 0.6 & 9 \\
\hline
\end{tabular}

Notes: ${ }^{a}$ Data was calculated on 512 patients as this data was not available for 13 patients. 


\section{Patients and Clinical Centers per month}

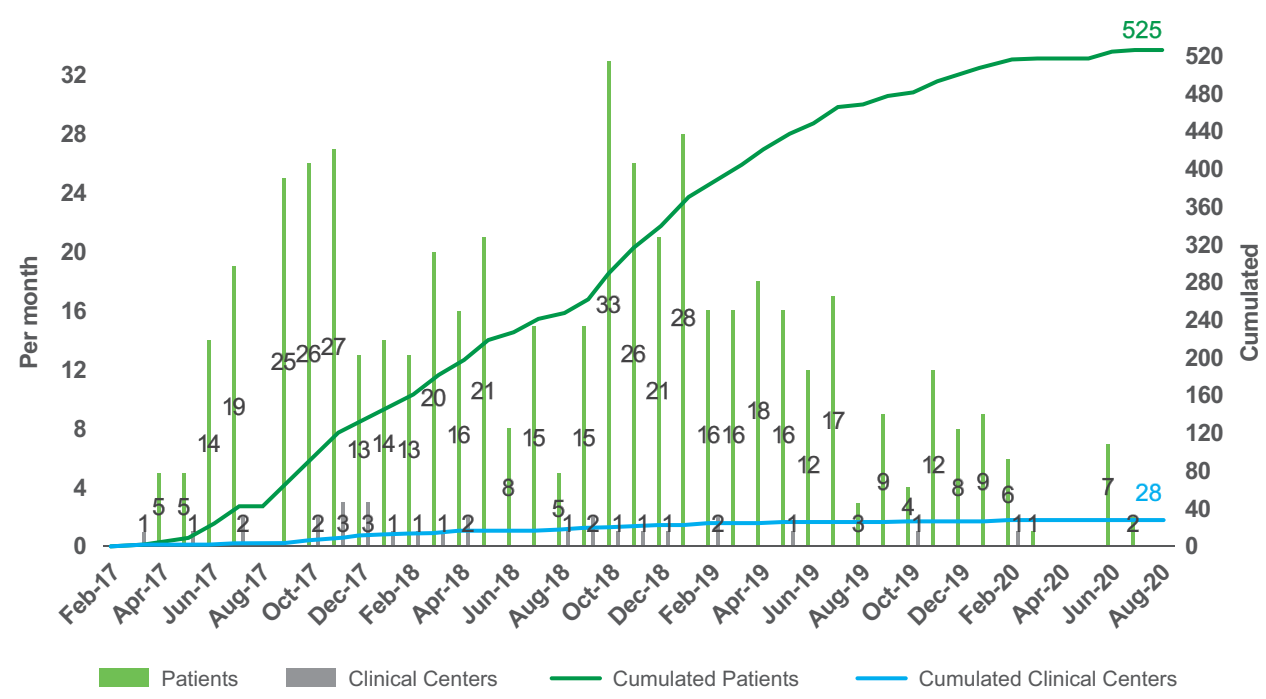

Figure 2 Uptake of patients and Clinical Centers into the PSOLife CARE Program.

Centers proposed the program nearer to the prescription date.

Throughout the 42-month Program $>1300$ specialist sessions were executed, including 1135 outpatient visits to a Dermatologist, 188 visits to a Psychologist, 53 visits by a field Nurse, and 18 visits to a Nutritionist. The increase in the number of visits was linked to the increase in the number of patients enrolling in the Program. Patients also received support, education, and training via phone calls, with a total of 6934 remote sessions implemented.

During the Program, 3340 administrations of secukinumab were recorded. Of these, 3066 (92\%) were administered in an acceptable range (1137 administered between 0-9 days with an average duration of 7 days, and, 1929 administered between 24-34 days with an average duration of 29 days). Conversely, there were 274 administrations in an anomalous range (67 administered between 10-23 days with an average duration of 18 days, and, 207 administered $\geq 35$ days with an average duration of 44 days).

\section{Patient Satisfaction}

Across the PSOLife CARE Program treatment period, patient satisfaction was high with an average score per 6-month period between 4.0 ("Very satisfactory") and 4.8 (almost "Outstanding") (Figure 3). Patient satisfaction with the PSP declined from October 2019 to March
2020 before recovering from April 2020 to September 2020.

For the patient satisfaction questionnaire, 680 responses were collected. Most patients felt they had received clear and complete information that allowed them to easily activate the Program services, with $29.6 \%$ (201/680), 8.8\% (60/680), and 60.9\% (414/680), respectively, of patients rating the Program as "Satisfactory", "Very satisfactory", and "Outstanding". When asked whether the Program had improved their confidence in drug administration and management and whether they found the Program helpful in following the recommendations provided by the Center where they were being treated, $98.1 \%$ of patients (667/680) rated these items as "Satisfactory" to "Outstanding".

Overall, the quality of the Program was rated as "Very satisfactory" to "Outstanding" by $86.3 \%$ of patients (587/ 680) and most patients felt supported by the PSOLife CARE Program when dealing with the management of their psoriasis, with 23.4\% (159/680), 14.7\% (100/680), and 59.9\% (407/680) of patients, respectively, rating the Program as "Satisfactory", "Very satisfactory", and "Outstanding". Only 31 (4.6\%) patients stated that the Program should be changed in some way, specifically with regards to some of the services provided within the Program.

The emotional, social, physical, and economic impact of psoriasis on quality of life over a 24-month detection period is shown in Figure 4A-D. The impact of psoriasis on all four aspects decreased after the first month in the 


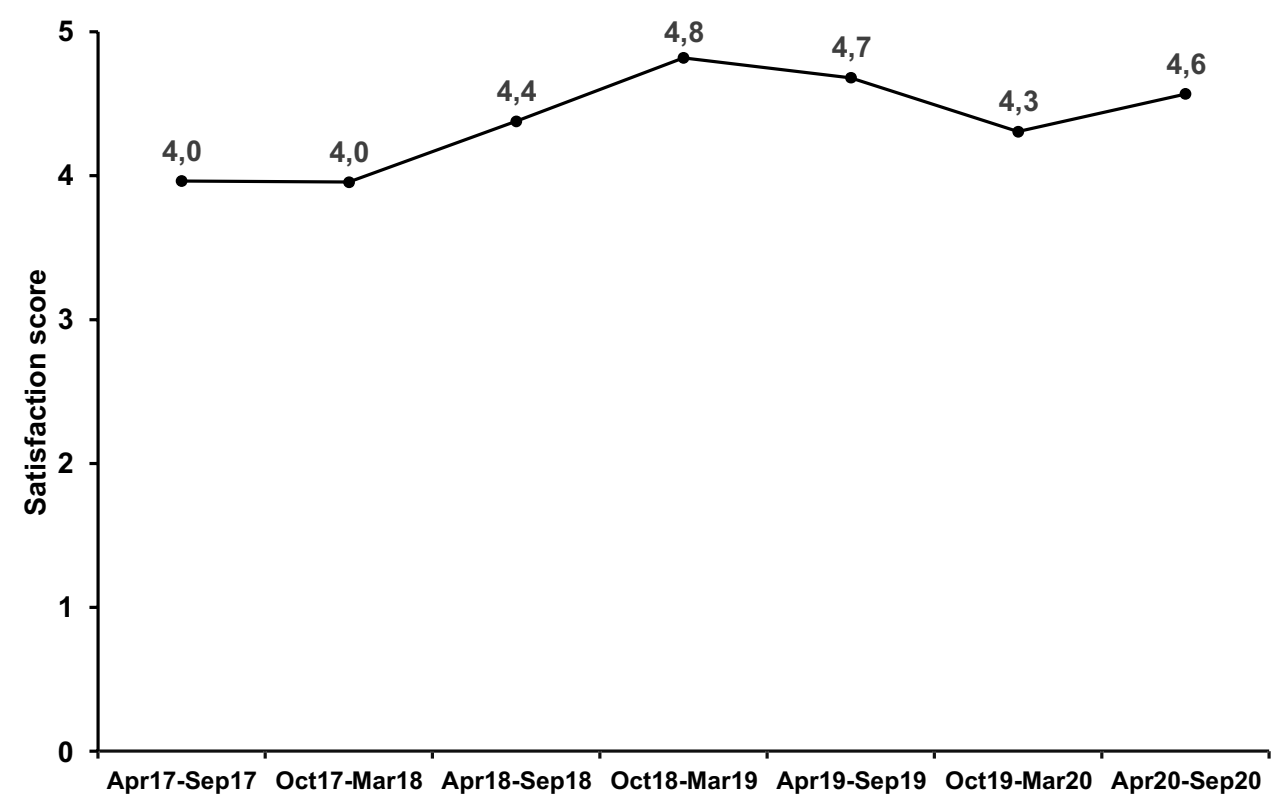

$\rightarrow$ Average score

Figure 3 Overall patient satisfaction with the PSOLife CARE Program per treatment period.

Notes: Satisfaction score: I - Did not meet my expectations; 2 - Fairly Satisfactory; 3 - Satisfactory; 4 - Very Satisfactory; 5 - Outstanding.
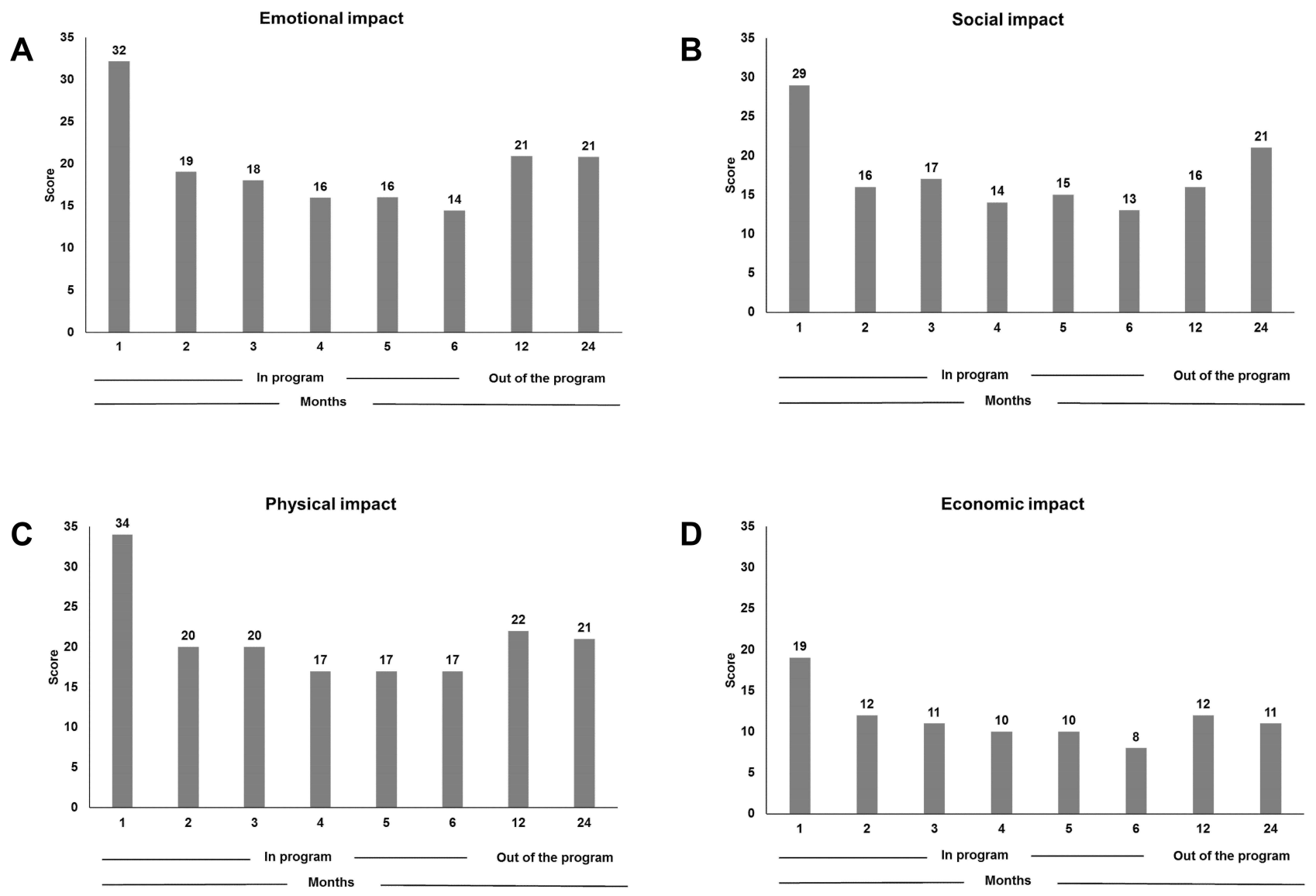

Figure 4 The (A) emotional, (B) social, (C) physical, and (D) economic impact of psoriasis on patients' quality of life over a 24-month detection period during the PSOLife CARE Program. 
PSOLife CARE Program and remained relatively stable over time.

\section{Medical Professional Satisfaction}

Medical professionals were also surveyed on their satisfaction with the PSOLife CARE Program and asked to evaluate the impact of the Program on their approach with patients. Of the 81 medical professionals with patients who had $>6$ months of stay in the Program, 28 Dermatologists responded. When asked whether they felt the Program was supportive in their management of patients treated with secukinumab, 25.0\% (7/28), 39.3\% $(11 / 28)$, and $35.7 \%(10 / 28)$, respectively, of medical professionals reported this as being "Satisfactory", "Very satisfactory", and "Outstanding".

All medical professionals who responded to the survey felt their patients were better monitored and better informed of their disease, especially regarding the management of secukinumab (54\%), the dermatological aspect $(21 \%)$, and the psychological aspect $(14 \%)$ of their psoriasis, as well as the side effects associated with secukinumab (11\%). The majority of medical professionals (71\%) felt the monitoring visits were not taking less time, with time saved on average at each visit equaling 5-10 minutes,

A

After 6 months out of the Program, in which of the following aspects do you think the PSOLife Program's calls have been helpful?

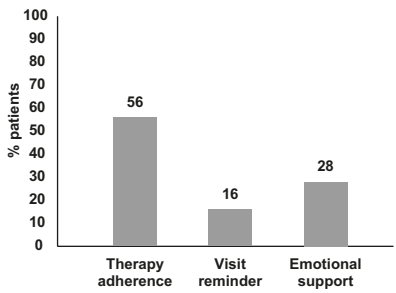

$\mathrm{E}$

After 12 months out of the Program, in which of the following aspects do you think the PSOLife Program's calls have been helpful?
callo

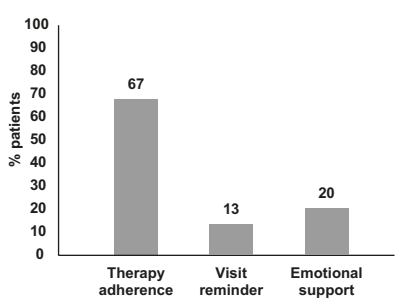

B

How do you consider/assess your therapy adherence since you are no longer under the Program?

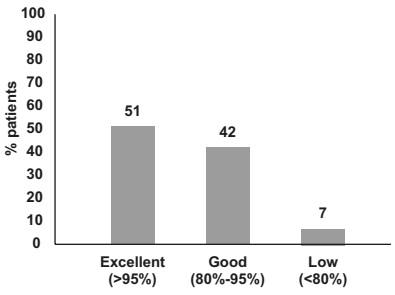

$\mathbf{F}$

How do you consider/assess your therapy adherence since you are no longer under the Program?

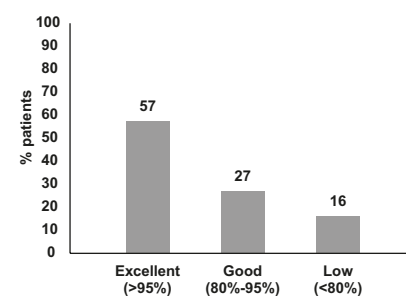

4-1 minutes, and 0 minutes, respectively, for $23 \%, 9 \%$, and $68 \%$ of medical professionals.

More than half $(57 \%)$ of medical professionals reported that they were able to get more information about their patients' disease management, specifically regarding patient adherence (32\%) and compliance with recommendations $(29 \%)$. When asked their opinion on the overall quality of the Program, 53.6\% rated it as "Outstanding", $32.1 \%$ as "Very satisfactory", and 14.3\% as "Satisfactory".

\section{Treatment Adherence}

Most patients continued to adhere to treatment despite discontinuing the Program, with discontinuation of patients after 6-months being due to the structure of the Program (Figures $5 \mathrm{~A}$ and E). At 12 months, $51 \%$ of patients $(n=82)$ considered their therapy adherence to be excellent ( $>95 \%)$, $42 \%$ considered their therapy adherence to be $\operatorname{good}(>80 \%$ to $<95 \%$ ), whereas treatment adherence was low $(<80 \%)$ for $7 \%$ of the patients (Figure $5 \mathrm{~B})$; at 24 months $(n=182)$, these figures were $57 \%, 27 \%$, and 16\%, respectively (Figure 5F). Almost three-quarters of patients $(71 \%$ and $73 \%$, respectively) continued to receive secukinumab at 12 and 24 months (Figures 5C and $\mathrm{G}$ ); the main motivation for

C Are you still in treatment
with secukinumab?

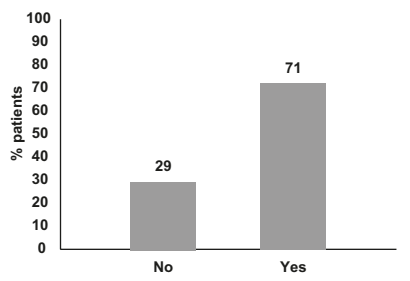

G

Are you still in treatment with secukinumab?

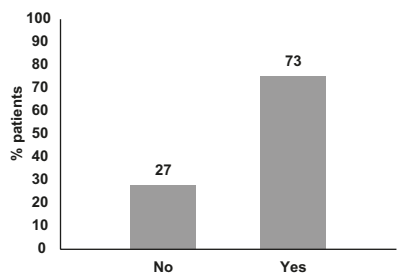

D

If not in treatment specify the motivation.

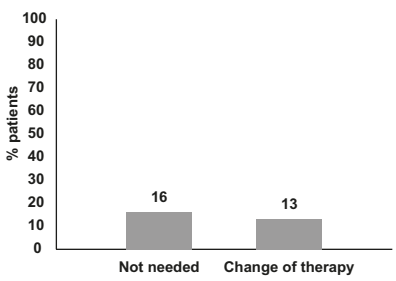

H

If not in treatment, specify the motivation.

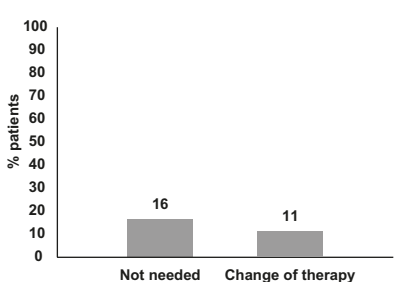

Figure 5 Treatment adherence at $(\mathbf{A}-\mathbf{D}) 12$ months $(n=82)$ and $(\mathbf{E}-\mathbf{H}) 24$ months $(n=182)$. 
treatment discontinuation was that it was no longer needed (16\% at both 12 and 24 months) (Figures 5D and $\mathrm{H}$ ).

It should be noted, however, that this survey was implemented towards the end of 2019 and therefore after the PSP had started. Consequently, we were only able to reach some of the patients, which explains why the patient numbers are low. We were unable to contact all patients after their exit from the Program for the following reasons: unavailability of some patients after leaving (no answer to telephone calls); some patients were immediately subjected to the questionnaire as they had left the PSP sometime prior, which explains the higher patient number at 24 months $(\mathrm{n}=182)$ than at 12 months $(\mathrm{n}=82)$.

\section{Discussion}

Overall, patient satisfaction remained consistently high during the PSOLife CARE Program, with sustained improvements observed in all aspects of quality of life (ie emotional, social, physical, and economic). Adherence to secukinumab continued over the long-term for most patients despite exiting from the Program. Medical Professionals also reported positive outcomes on their interactions with patients, with more than half of those surveyed rating the overall quality of the Program as "Outstanding".

Medication adherence is a key component of a successful treatment regimen. By supporting patient adherence, the PSOLife CARE Program may have empowered patients to better manage their psoriasis. This is likely to be a direct result of the high number of outpatient visits undertaken, the majority of which involved a Dermatologist consultation, and of the remote sessions offering comprehensive specialty support, education, and training to patients via phone calls.

The PSP captures the patients' need to be followed during the treatment once the specialist dermatologist has set the therapy. Personalized support accompanies patients over time, making them more solid, autonomous, aware, and adherent to treatment. In addition, the PSP PSOLife CARE Program has a holistic approach to the patient because it offers support on other comorbidities that can occur together with psoriasis, for which it is essential to have multidisciplinary management of the disease (this is the reason for the visits with a psychologist and nutritionist). All this can only have a positive impact on the patient and their quality of life in general.

To achieve therapeutic goals in psoriasis, treatment adherence and persistence are crucial. Patients in the real-world often discontinue treatment due to loss of effectiveness or the development of adverse side effects, lowering the rates of drug survival compared with clinical trials. ${ }^{21-23}$ Participation in PSPs, such as the PSOLife CARE Program, may add significant value to the treatment of patients with psoriasis by improving their satisfaction with treatment, treatment adherence, and quality of life.

Approximately two-thirds of patients rated the quality of the PSOLife CARE Program as "Outstanding" and felt that they had received clear and complete information that allowed them to easily activate the Program services. Importantly, most patients felt that the Program helped them to manage and administer secukinumab correctly. This has important implications on maintaining adherence to treatment over the long-term, with $57 \%$ of patients rating their adherence as excellent despite having been out of the Program for 12 months. In the SCULPTURE Extension study, high levels of skin clearance were sustained through 5 years of treatment with secukinumab and associated with improved health-related quality of life in patients with moderate-to-severe psoriasis. ${ }^{9}$ Hence, improvement in the quality and effectiveness of care, and consequently improved confidence in managing their psoriasis, could lead to long-lasting skin clearance and a better quality of life for patients.

PSPs support better disease management in chronic autoimmune diseases. The HUMIRA Complete PSP, for example, provided one-on-one, personal support to patients with chronic autoimmune diseases who were receiving adalimumab. ${ }^{18-20}$ Over a 12-month follow-up period, participation in the HUMIRA Complete PSP was associated with improved adherence and persistence to adalimumab, significantly lowering medical costs and overall health care costs. In a systematic review of 64 trials or observational studies of PSPs targeting chronic diseases, at least one significant positive clinical outcome was reported in $41(64.1 \%)$ studies. $^{24}$ Whilst a positive outcome on adherence was the most commonly reported clinical outcome, the review also found positive impacts on clinical and humanistic outcomes, including functional status and quality of life, as well as reduced health care utilization and costs. Consequently, through the use of PSPs, pharmaceutical companies can augment therapeutic strategies and improve patient outcomes. Health care systems are also increasingly recognizing the value of PSPs due to lowered health care costs.

\section{Limitations}

There are limitations to the PSOLife CARE Program that must be acknowledged. None of the questionnaires used to 
assess outcomes were validated, questioning the accuracy of their results. The length of the detection period, with a standard duration in the Program of only 6 months, may not have been long enough for habits to be established as routine. As we did not limit data analysis to patients who had concluded the PSP by August 2020, and data accumulation is ongoing, some patients may not have completed their 6 month stay and therefore represent an "incomplete" path. Moreover, the Program was not designed as a clinical, randomized, or observational study and, as such, there were no controls on the patient population. This may have resulted in unknown biases and skewed outcomes.

It should also be acknowledged that the lockdown restrictions associated with the COVID-19 pandemic may have impacted the PSOLife CARE Program as there was a noticeable decrease in the uptake of patients into the Program from February 2020, although patient satisfaction scores remained high from April 2020 to September 2020. Indeed, the COVID-19 pandemic was unlikely to have affected the patient satisfaction data linked to the PSP because patients could still be contacted through the remote contact center.

\section{Conclusion}

With improved adherence to secukinumab, both patients and physicians were satisfied by the PSOLife CARE Program. Ultimately, PSPs aim to enhance the benefits, value, and quality of patient care, improve treatment management, and increase long-term adherence to therapy. The benefits of PSPs can be observed for patients, caregivers, and medical professionals, and may bring about economic benefits to health care systems, such as the Italian National Health Services, attributable to decreased health care utilization and costs for well-managed patients.

\section{Acknowledgments}

The PSOLife CARE Patient Support Program and medical writing assistance were financially supported by Novartis. The Authors would like to acknowledge the invaluable input of their collaborators (Dermatological, Nutritional, and Psychological) who executed the visits during the program. In paticular Ambrogio Francesca, Babino Graziella, Baione Margherita, Bartolotta Annunziata, Battimello Valeria, Benintende Clara, Bonatti Chiara, Castelli Alessandro, D'Agostino Alessandra, D'Antonio Alessandra, De Rosa Alina, Dealessi Valeria, Di Costanzo Luisa, Fidanza Rosaria, Gambardella Alessio, Galluzzo Marco, Genovese Paola, Graziola Francesca,
Javor Sanja, Lembo Claudio, Loiacono Anna, Macaluso Laura, Marullo Marialuisa, Massari Monica, Miglino Benedetta, Moretta Gaia, Panarese Fabrizio, Petralia Maria Cristina, Presciutti Maria Beatrice, Quartarone Palma, Ramondetta Alice, Riso Gabriella, Romita Paolo, Ruggiero Marta, Ruotolo Monica, Tambone Sara, Scuderi Roberta, Torti Sonia, Tarantino Vanessa, and Vozza Giuseppe. Medical writing assistance was provided by Melanie Gatt $(\mathrm{PhD})$, an independent medical writer on behalf of Springer Healthcare.

\section{Disclosure}

Giuseppe Argenziano has no conflict of interest to declare. Paolo Amerio has served as a consultant or Speaker for Abbvie, Celgene, Pfizer, Sanofi-Genzyme, Eli Lilly, and Jannsen, Galderma. Maria Grazia Aragone has served on advisory boards for Novartis, AbbVie, LeoPharma. Ginevra Baggini has no conflict of interest to declare. Manuela Baldari has no conflict of interest to declare. Gianpiero Castelli has no conflict of interest to declare. Simmaco Coppola is IQVIA employee. Antonio Costanzo has served as an advisory board member and consultant, and has received fees and speaker's honoraria or has participated in clinical trials for Abbvie, Almirall, Biogen, LEO Pharma, Lilly, Janssen, Novartis, Pfizer, Sanofi Genzyme, and UCB-Pharma. De Pasquale has no conflict of interest to declare. Maria Concetta Fargnoli has served on advisory boards, received honoraria for lectures and research grants from Mylan, Medac Pharma, Pierre Fabre, Sanofi-Genzyme, Sunpharma, MSD. Caterina Foti advisory board and speaker for Novartis, Sanofi, Abbvie, Lillie. Claudia Giofrè has been a member of scientific board/speaker/clinical study investigator for AbbVie, Celgene, Janssen, Novartis, Sanofi. Serena Lembo has no conflict of interest to declare. Claudia Leporati has served on advisory boards for Novartis, AbbVie, Leo Pharma, Almirall. Francesco Loconsole served on advisory boards and/or received honoraria for lectures from Abbvie, Jansen-Cilag, Novartis, Lilly, Sanofi. Giovanna Malara has been a member of scientific board/speaker/clinical study investigator for AbbVie, Celgene, Janssen, Eli-Lilly, Novartis, Sanofi. Ketty Peris reports personal fees from Abbvie, personal fees from Almirall, personal fees from Lilly, personal fees from Galderma, personal fees from Leo Pharma, personal fees from Pierre Fabre, personal fees from Novartis, personal fees from Sanofi, personal fees from Sun Pharma, personal fees from Janssen, outside the submitted work. Antonio Giovanni Richetta has been a member of scientific board/speaker/clinical study investigator for AbbVie, Celgene, Janssen, Eli-Lilly, Novartis. Paola Savoia has no 
conflict of interest to declare. Rossana Tiberio has no conflict of interest to declare. Massimo Travaglini has no conflict of interest to declare. Maria Teresa Uzzauto has no conflict of interest to declare. Luca Bianchi has been speaker or board member for Abbie, Novartis, Eli Lilly, Biogen, Sanofi, Leo Pharma, Janssen. The authors report no other conflicts of interest in this work.

\section{References}

1. Di Meglio P, Villanova F, Nestle FO. Psoriasis. Cold Spring Harb Perspect Med. 2014;4:a015354. doi:10.1101/cshperspect.a015354

2. Prignano F, Rogai V, Cavallucci E, Bitossi A, Hammen V, Cantini F. Epidemiology of psoriasis and psoriatic arthritis in Italy-A systematic review. Curr Rheumatol Rep. 2018;20:43. doi:10.1007/s11926-0180753-1

3. Saraceno R, Mannheimer R, Chimenti S. Regional distribution of psoriasis in Italy. J Eur Acad Dermatol Venereol. 2008;22:324-329.

4. Valent F, Tullio A, Errichetti E, Stinco G. The epidemiology of psoriasis in an Italian area: population-based analysis of administrative data. G Ital Dermatol Venereol. 2020;155:652-657. doi:10.23736/S0392-0488.18.06020-0

5. Vena GA, Altomare G, Ayala F, et al. Incidence of psoriasis and association with comorbidities in Italy: a 5-year observational study from a national primary care database. Eur J Dermatol. 2010;20:593-598.

6. Griffiths CEM, Jo SJ, Naldi L, et al. A multidimensional assessment of the burden of psoriasis: results from a multinational dermatologist and patient survey. Br J Dermatol. 2018;179:173-181. doi:10.1111/bjd.16332

7. Rendon A, Schakel K. Psoriasis pathogenesis and treatment. Int $J$ Mol Sci. 2019;20:1475. doi:10.3390/ijms20061475

8. Radi G, Campanati A, Diotallevi F, Bianchelli T, Offidani A. Novel therapeutic approaches and targets for treatment of psoriasis. Curr Pharm Biotechnol. 2021;22:7-31. doi:10.2174/13892010216662 00629150231

9. Bissonnette R, Luger T, Thaci D, et al. Secukinumab demonstrates high sustained efficacy and a favourable safety profile in patients with moderate-to-severe psoriasis through 5 years of treatment (SCULPTURE Extension Study). J Eur Acad Dermatol Venereol. 2018;32:1507-1514.

10. Bissonnette R, Luger T, Thaci D, et al. Secukinumab sustains good efficacy and favourable safety in moderate-to-severe psoriasis after up to 3 years of treatment: results from a double-blind extension study. Br J Dermatol. 2017;177:1033-1042. doi:10.1111/bjd.15706

11. Blauvelt A, Reich K, Tsai TF, et al. Secukinumab is superior to ustekinumab in clearing skin of subjects with moderate-to-severe plaque psoriasis up to 1 year: results from the CLEAR study. $J \mathrm{Am}$ Acad Dermatol. 2017;76:60-69 e69. doi:10.1016/j.jaad.2016.08.008

12. Deodhar A, Mease PJ, McInnes IB, et al. Long-term safety of secukinumab in patients with moderate-to-severe plaque psoriasis, psoriatic arthritis, and ankylosing spondylitis: integrated pooled clinical trial and post-marketing surveillance data. Arthritis Res Ther. 2019;21:111. doi:10.1186/s13075-019-1882-2

Patient Preference and Adherence

\section{Publish your work in this journal}

Patient Preference and Adherence is an international, peer-reviewed, open access journal that focusing on the growing importance of patient preference and adherence throughout the therapeutic continuum. Patient satisfaction, acceptability, quality of life, compliance, persistence and their role in developing new therapeutic modalities and compounds to optimize clinical outcomes for existing disease
13. Langley RG, Elewski BE, Lebwohl M, et al. Secukinumab in plaque psoriasis-results of two Phase 3 trials. $N$ Engl J Med. 2014;371:326338. doi:10.1056/NEJMoa1314258

14. Mrowietz U, Leonardi CL, Girolomoni G, et al. Secukinumab retreatmentas-needed versus fixed-interval maintenance regimen for moderate to severe plaque psoriasis: a randomized, double-blind, noninferiority trial (SCULPTURE). J Am Acad Dermatol. 2015;73:27-36 e21. doi:10.1016/j. jaad.2015.04.011

15. Bagel J, Blauvelt A, Nia J, et al. Secukinumab maintains superiority over ustekinumab in clearing skin and improving quality of life in patients with moderate to severe plaque psoriasis: 52-week results from a double-blind phase $3 \mathrm{~b}$ trial (CLARITY). J Eur Acad Dermatol Venereol. 2020;35:135-142. doi:10.1111/jdv.16558

16. European Medicines Agency. Cosentyx (secukinumab). Available from: https://www.ema.europa.eu/en/medicines/human/EPAR/cosen tyx. Accessed October 7, 2020.

17. Novartis. COSENTYX ${ }^{\circledR}$ (secukinumab) for injection, for subcutaneous use: US prescribing information; 2020. Available from: https://www.novartis.us/sites/www.novartis.us/files/cosentyx.pdf. Accessed October 7, 2020.

18. Brixner D, Mittal M, Rubin DT, et al. Participation in an innovative patient support program reduces prescription abandonment for Adalimumab-treated patients in a commercial population. Patient Prefer Adherence. 2019;13:1545-1556. doi:10.2147/PPA. S215037

19. Brixner D, Rubin DT, Mease P, et al. Patient support program increased medication adherence with lower total health care costs despite increased drug spending. J Manag Care Spec Pharm. 2019;25:770-779.

20. Rubin DT, Mittal M, Davis M, Johnson S, Chao J, Skup M. Impact of a patient support program on patient adherence to adalimumab and direct medical costs in crohn's disease, ulcerative colitis, rheumatoid arthritis, psoriasis, psoriatic arthritis, and ankylosing spondylitis. $J$ Manag Care Spec Pharm. 2017;23:859-867.

21. Georgakopoulos JR, Ighani A, Phung M, Yeung J. Drug survival of secukinumab in real-world plaque psoriasis patients: a 52-week, multicenter, retrospective study. $J$ Am Acad Dermatol. 2018;78:1019-1020. doi:10.1016/j.jaad.2017.11.036

22. Torres T, Balato A, Conrad C, et al. Secukinumab drug survival in patients with psoriasis: a multicenter, real-world, retrospective study. $J$ Am Acad Dermatol. 2019;81:273-275. doi:10.1016/j.jaad.2019.02.031

23. Yiu ZZN, Mason KJ, Hampton PJ, et al. Drug survival of Adalimumab, ustekinumab and secukinumab in patients with psoriasis: a prospective cohort study from the British Association of Dermatologists Biologics and Immunomodulators Register (BADBIR). $\mathrm{Br} J$ Dermatol. 2020;183:294-302. doi:10.1111/bjd.18981

24. Ganguli A, Clewell J, Shillington AC. The impact of patient support programs on adherence, clinical, humanistic, and economic patient outcomes: a targeted systematic review. Patient Prefer Adherence. 2016;10:711-725. states are major areas of interest for the journal. This journal has been accepted for indexing on PubMed Central. The manuscript management system is completely online and includes a very quick and fair peer-review system, which is all easy to use. Visit http:// www.dovepress.com/testimonials.php to read real quotes from published authors.

\section{Dovepress}

